\title{
Transbronchial biopsy as a tool to evaluate small airways in asthma
}

\author{
S. Balzar, S.E. Wenzel, H.W. Chu
}

Transbronchial biopsy as a tool to evaluate small airways in asthma. S. Balzar, S.E. Wenzel, H.W. Chu. (C) ERS Journals Ltd 2002.

ABSTRACT: Small airway (SA) inflammation in asthmatics is poorly understood. Surgical biopsies to obtain peripheral lung tissue are seldom justified in asthmatics. Therefore, the authors hypothesised that transbronchial biopsy could be an alternative approach to evaluate $\mathrm{SA}$ in asthma.

Transbronchial and endobronchial biopsy tissue samples (TBBX and EBBX) from 12 severe asthmatics were evaluated for airway and parenchymal total inflammatory cell count expressed as the sum of immunostained T-cells (CD3), macrophages (CD68), mast cells (tryptase AA1), neutrophils (neutrophil elastase) and eosinophils (EG2) per $\mathrm{mm}^{2}$. The large airways (LA) were evaluated in EBBXs, while SA, medium airways (MA) and alveolar tissue (AT) were evaluated in TBBXs.

When cell counts from SA, MA, LA and AT were compared, SA had a significantly higher cell count than MA or LA (SA $1011 \cdot \mathrm{mm}^{-2}(539-1,290)$, MA $346 \cdot \mathrm{mm}^{-2}$ (223-415), LA $332 \cdot \mathrm{mm}^{-2}(189-416)$, AT $\left.464 \cdot \mathrm{mm}^{-2}(298-834)\right)$. The cell density and pattern of the inflammatory cell distribution in subjects with TBBXs appeared similar to those in three severe asthmatics whose inflammatory cells were analysed in surgical tissue samples.

This study suggests that small airway may be identified and analysed in transbronchial biopsy tissue samples and therefore transbronchial biopsy tissue samples could expand the analysis of inflammation and tissue remodelling in asthma.

Eur Respir J 2002; 20: 254-259.
National Jewish Medical and Research Centre, the University of Colorado Health Sciences Center, Denver, CO, USA.

Correspondence: S. Balzar, National Jewish Medical and Research Center, 1400 Jackson St., D 203, Denver, CO 80206, USA.

Fax: 13033981851

E-mail: balzars@njc.org

Keywords: Asthma/pathology asthma/inflammation

bronchoscopy

Received: July 182001

Accepted after revision: April 32002

This study was supported by grant AI40600 and American Lung Association of Colorado and Oklahoma, USA.
Airway inflammation is a predominant element of asthma pathology. Although differences in morphometric approaches exist, analysis of endobronchial biopsy tissue obtained through the flexible fiberoptic bronchoscope has confirmed the presence of inflammatory cells in the large airways (LA) from newly diagnosed asthma patients, as well as from severe patients on high doses of anti-inflammatory medications $[1,2]$.

Physiological and pathological studies suggest that inflammation in asthma is present in the small airways (SA), and perhaps the lung parenchyma [2-9]. Unfortunately, these distal regions of the lung have been difficult to evaluate due to the invasiveness of the open lung/thoracoscopic biopsy procedure required to obtain tissue. Transbronchial biopsy tissue samples (TBBX) have been traditionally used in interstitial lung diseases for pathological diagnoses, often based on descriptive pathological assessment alone [10-13]. While morphometric analysis of alveolar tissue (AT) (only) in TBBX has been reported [2, 5, 9, 14, 15], morphometric analysis of airway tissue in TBBX, as a separate lung tissue compartment has not been performed.

The authors hypothesised that SA tissue can be sampled in TBBXs and that the density of the inflammatory cell infiltrate in severe asthmatics' SAs

For editorial comments see pages 247 and 249. will be higher than in the LAs. Therefore, the total number of inflammatory cells per area of tissue was chosen as the primary parameter to follow through the central and peripheral airways and in the lung parenchyma of severe asthmatics. Additionally, the total numbers of inflammatory cells in the airways and parenchyma of subjects with TBBX were evaluated in association with data from three severe asthmatics whose SA inflammation could be characterised from multiple complete airways from surgically obtained lung tissue samples.

\section{Material and methods}

\section{Tissue acquisition}

Concurrent endobronchial biopsy tissue samples (EBBXs) and TBBXs were obtained from 12 severe, steroid-dependent asthma patients referred to the National Jewish Medical and Research Center for evaluation and treatment of their disease. Bronchoscopies were performed as described previously $[2,16]$. Briefly, subjects were premedicated with atropine $(0.6 \mathrm{mg})$ and codeine $(30-60 \mathrm{mg})$ intramuscularly, and nebulised albuterol. Lidocaine $(<400 \mathrm{mg}$ total $)$ was used as a local anaesthetic for upper and lower airways. The bronchoscope was passed and endobronchial biopsies performed at the carina under direct 
visualisation (usually from the third to fourth airway), followed by transbronchial biopsies performed under fluoroscopic guidance. The transbronchial biopsies were done by passing the forceps (FB-15c-1; Olympus Inc., Melville, NY, USA or KA1811S; Pentax, Orangeburg, NY, USA) into a lateral subsegment of the left or right lower lobe, $1-2 \mathrm{~cm}$ from the fluoroscopically visualised lung perimeter. The forceps was then withdrawn $2-3 \mathrm{~cm}$, the cup opened and the forceps re-advanced one-half the original distance. Two to four TBBX were obtained per subject. The subjects received oxygen and vital signs were monitored throughout the procedure.

In the group of subjects with surgical tissue samples (STSs), peripheral lung tissue samples were obtained by thoracoscopic/open lung biopsy from three severe asthmatics refractory to high doses of oral steroids and standard asthma medications and studied for clinical reasons to rule out other diagnoses, of which none were found. For two of these subjects concurrent EBBXs were available for analysis. One subject underwent endobronchial, transbronchial and open lung biopsy on the same day. The tissue from this subject was included in both the TBBX group (the subject's EBBX and TBBX) and the STS group (the subject's STS).

This study was approved by the Institutional Review Board of the National Jewish Medical and Research Center. All subjects gave informed consent.

\section{Tissue processing}

TBBXs and EBBXs were acetone fixed (at $-20^{\circ} \mathrm{C}$ overnight) and embedded in glycol methacrylate (GMA) resin [9]. Tissue blocks were stored at $-20^{\circ} \mathrm{C}$ in airtight containers with dessicant.

STSs from two subjects were fixed in acetone and embedded in GMA as described previously. The third subject's STSs were fixed in $10 \%$ formalin and embedded in paraffin.

\section{Airway classification}

Small airways (SA) in TBBX were defined as airway tissue without cartilage and/or mucous glands, which had AT attached to it. Small airways in STS were defined as membranous airways with a subepithelial basement membrane perimeter of $<6 \mathrm{~mm}$, in the absence of cartilage and/or mucous glands in the airway wall [6]. Figure 1 shows typical small airways from TBBX and STS.

Medium airways (MA) in TBBX were defined as airway tissue that contained cartilage and/or mucous glands and distal from the third to fourth generation airways.

Large airways (LA) were defined as airway tissue obtained through endobronchial biopsy, usually from the third to fourth generation airways.

\section{Immunostaining}

Serial $2 \mu \mathrm{m}$ sections were cut from well-oriented tissue blocks. Immunostaining was performed as reported previously using Vectastain Elite ABC Kits (Vector Laboratories, Burlingame, CA, USA) [17]. Tissue sections were immunostained for the T-cell marker CD3, monocyte/macrophage marker CD68, mast cell tryptase AA1, neutrophil elastase (all from Dako, Carpinteria, CA, USA) and eosinophil cationic protein EG2 (Pharmacia, Uppsala, Sweden). Figure 2 shows an example of mast cell tryptase immunostaining in TBBX and STS.

\section{Morphometric analysis}

Positively stained nucleated cells in LA, MA and SA were counted in the area between the subepithelial basement membrane and the smooth muscle layer, excluding blood vessels, mucous glands and areas of bleeding. A smooth muscle layer was observed in all SA. The cell count in the AT was analysed only in TBBX tissue containing SA, with AT attached to the SA wall. AT cell counts in STS were analysed in the area surrounding SA, excluding areas of damage and bleeding. To obtain cell density, cell numbers were normalised per area of tissue analysed. The area of tissue was measured using computerised imaging system and Scion Image software (National Institute of Health).

The total inflammatory cell count was expressed as the sum of all cell types $\cdot \mathrm{mm}^{-2}$.

The coefficient of variation in cell counts by the same observer or two different observers for the same sections was $\leqslant 7 \%$.

\section{Statistical analysis}

Overall comparisons of data from different lung regions were analysed initially using the nonparametric Kruskal-Wallis variation of the Wilcoxon test. If a significant difference existed $(p<0.05)$, the TukeyKramer test was used to identify statistically significant differences between the different lung regions [18].

\section{Results}

\section{Subject characteristics}

The 14 subjects studied were severe asthmatics with difficult-to-control asthma, requiring frequent urgent or emergent healthcare. These asthmatics met published criteria for severe disease [2, 16]. They had a forced expiratory volume in one second (FEV1) of $<70 \%$ of predicted, with at least a $15 \%$ improvement in FEV1 postbronchodilator. Along with other asthma medications (long acting $\beta$-agonists, leukotriene modulators, theophylline and short-acting $\beta$-agonists), they were taking $>10 \mathrm{mg} \cdot \mathrm{day}^{-1}$ of prednisone or equivalent for at least $75 \%$ of the previous year. Eleven subjects were on oral steroid therapy, eight of them also using moderate-to-high doses of inhaled steroids. The remaining three subjects were maintained on high doses of inhaled steroids only, 

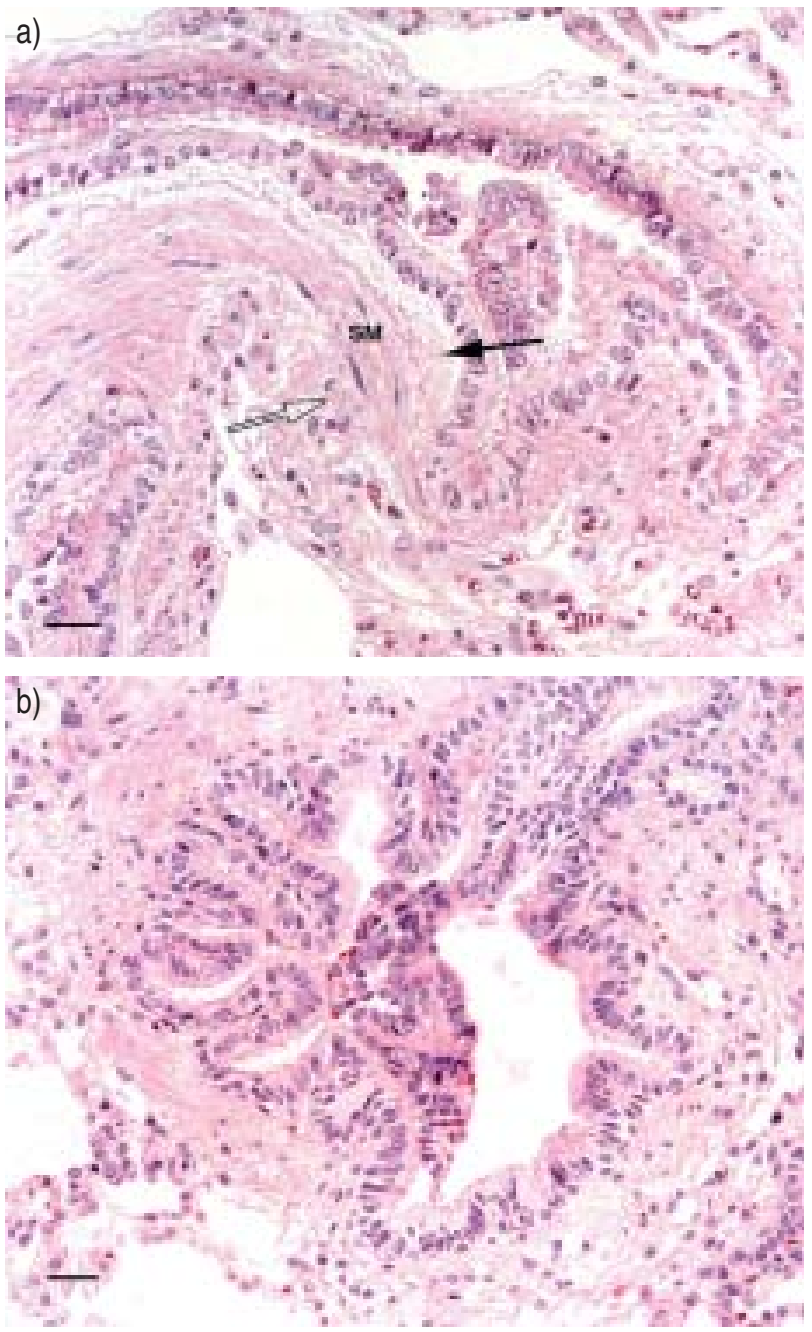

Fig. 1.-Representative photomicrographs of small airways in a) transbronchial biopsy and in b) surgical tissue sample. Solid and open arrows indicate small airway inner wall and outer wall, respectively. Haematoxylin-eosin staining. SM: smooth muscle. Internal scale bar $=30 \mu \mathrm{m}$.

with frequent courses of oral steroids in the past year such that they were on them the majority of the time.

These subjects had no other underlying diseases and none were current smokers (three subjects were former smokers with 1, 3.4 and 5 pack-yrs, respectively). They represented a homogenous group in terms of sex (six females and eight males), age (median 38 yrs (interquartile range $32-43))$ and FEV1 $(46 \%$ (37-56)).

\section{Morphometric analysis}

Twenty-nine TBBXs from twelve subjects (average 2.4 (range 2-4) samples per subject) were examined for the presence of airway mucosa. There were 13 TBBXs $(45 \%)$ with airway tissue: eight $(28 \%)$ with SA and five $(17 \%)$ with MA. Seven subjects had SA tissue only, one subject had both SA and MA tissue and four subjects had MA tissue only.

As the sampling of the different lung sites was performed concurrently, the distributions of cells
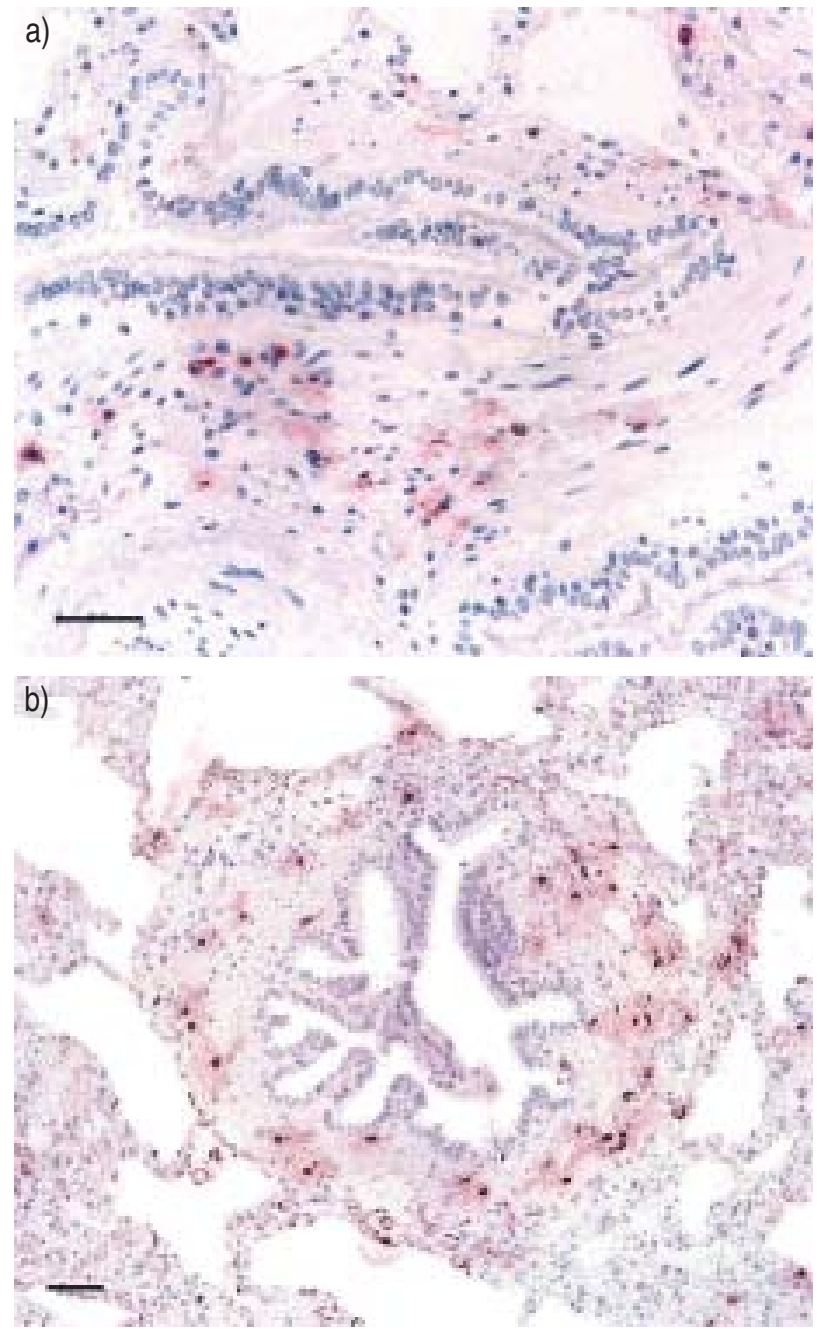

Fig. 2. - Mast cell tryptase immunostaining in small airways from a) transbronchial biopsy and from b) surgical tissue sample. Internal scale bar $=60 \mu \mathrm{m}$.

among the distinct lung regions were individually compared.

Distribution of inflammatory cell counts in medium airways, large airways, small airways and alveolar tissue. The distribution of inflammatory cell counts in MA, LA, SA and AT are shown in figure 3. The overall difference among inflammatory cell counts in the three airway sizes and AT was significant $(\mathrm{p}=0.008)$. The inflammatory cell count in SA (median $1011 \cdot \mathrm{mm}^{-2}$ (interquartile range 539-1290)) was significantly higher $(\mathrm{p}<0.05)$ than in MA $\left(346 \cdot \mathrm{mm}^{-2}(223-415)\right)$ and LA $\left(332 \cdot \mathrm{mm}^{-2}(189-416)\right)$, and not different $(\mathrm{p}=0.09)$ from AT $\left(464 \cdot \mathrm{mm}^{-2}(298-834)\right)$.

For the subject that underwent endobronchial, transbronchial and open lung biopsy on the same day, four SA and AT were evaluated in the STS and $\mathrm{MA}$ in TBBX. The counts of this subject followed the pattern of inflammatory cell distribution seen in both the TBBX and STS group, with a large difference between LA and SA cell counts $\left(89 \cdot \mathrm{mm}^{-2}\right.$ in LA versus $2777 \cdot \mathrm{mm}^{-2}$ in SA). The subject's MA cell 

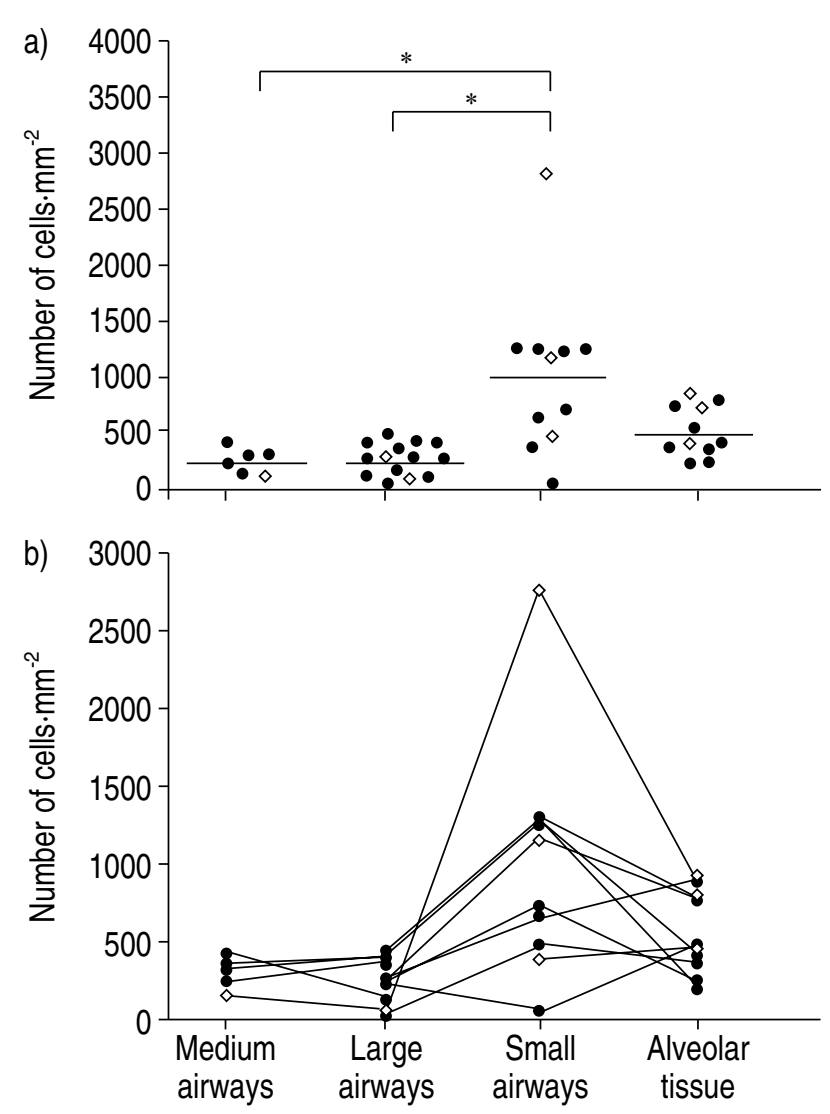

Fig. 3.-Inflammatory cell densities in severe asthmatics with transbronchial biopsy tissue samples (O) and surgical tissue samples $(\diamond)$. a) Inflammatory cell densities in small airways, medium airways, large airways and alveolar tissue. Crossbars represent medians. $*: \mathrm{p}<0.05$. Overall $\mathrm{p}=0.008$. b) Distribution of inflammatory cell counts throughout different lung regions as recorded in each study subject.

count $\left(181 \cdot \mathrm{mm}^{-2}\right)$ was similar to the LA count, while the AT cell count of $905 \cdot \mathrm{mm}^{-2}$ was similar to SA. A similar pattern of total cell counts was seen in every subject's tissue except one (fig. 3b).

As presented in table 1, the percentages of lymphocytes, mast cells and neutrophils were similar in all lung regions. The percentage of eosinophils was not statistically different, although only one subject had eosinophils in SA. The percentage of monocytes/ macrophages increased from proximal to distal lung and was significantly higher in AT than in LA $(\mathrm{p}=0.02)$.
Relationship of the transbronchial biopsy tissue samples data to the surgical tissue samples data. The relationship of the TBBX data to the STS data is shown in figure 3a. In the three subjects with STS, the SA morphometric analysis was performed in twelve complete SA cross-sections, with 2-6 SAs per subject. The cell count for each subject in that group was averaged. The inflammatory cell counts from the TBBXs for all airway sizes and AT overlap the data from the STS group of subjects. The small sample size of STS group limits statistical comparison.

\section{Discussion}

An increased awareness of the role of small airways in the pathophysiology of asthma requires that a suitable technique is developed to evaluate these peripheral airways in living asthmatics. A method that could balance risk and benefit in asthmatics of varying severity would be a significant step towards the exploration of peripheral lung changes. Thoracoscopic or open lung biopsy is, because of its invasiveness, rarely performed even in severe asthmatics. Transbronchial biopsy, although less invasive than surgical interventions, is not routinely performed in asthmatics, probably because of the potentially higher risk over endobronchial biopsy and the common perception that alveolar tissue pathological events are of minimal importance to the pathogenesis of asthma. However, the experience reported here with transbronchial biopsy tissue sampling suggests that, in addition to alveolar tissue, small airway tissue can be identified and evaluated in TBBXs.

This is the first transbronchial biopsy tissue study to morphometrically evaluate airway tissue found in TBBXs and to compare those inflammatory cell counts with large airways. Previous studies and the present authors own experience with small airways analysed in STS have suggested that high numbers of inflammatory cells may be present in small airways of asthmatics [3, 7, 19]. Therefore, inflammatory cell density was used in this study as an indicator of the difference between proximal and distal airways.

Although the airway's subepithelial basement membrane (internal) perimeter may be one of the most reliable parameters in defining a small airway, the internal perimeter cannot be used to define small airways in TBBX, as entire airway cross-sections are not available. Nevertheless, this study suggests that

Table 1.-Percentages of inflammatory cells in severe asthmatics with concurrent endobronchial and transbronchial biopsy

\begin{tabular}{lccccc}
\hline & Lymphocytes \% & Monocytes/macrophages \% & Mast cells \% & Neutrophils \% & Eosinophils \% \\
\hline Large airway & $22(15-44)$ & $16(13-28)$ & $14(6-22)$ & $33(19-47)$ & $1(0-8)$ \\
Medium airway & $11(4-24)$ & $26(23-31)$ & $26(15-39)$ & $9(4-45)$ & $0(0-29)$ \\
Small airway & $5(0-28)$ & $29(16-42)$ & $20(7-34)$ & $31(5-42)$ & $0(0-0)$ \\
Alveolar tissue & $4(0-19)$ & $40(32-47)$ & $20(15-27)$ & $29(21-43)$ & $3(0-5)$ \\
p-value & 0.2 & 0.02 & 0.22 & 0.68 & 0.08 \\
\hline
\end{tabular}

Data are presented as median (interquartile range) unless otherwise stated. ${ }^{\#}$ : p-value for comparison of the particular cell type percentages in all four lung regions. 
when the airway perimeter cannot be measured (as in TBBX), the histological characteristics may suffice to differentiate the two different kinds of airway tissue found in TBBXs. Small airways in TBBX resemble membranous bronchioles, lacking cartilage and/or mucous glands and with alveolar tissue attached to airway wall. Conversely, medium airways in TBBX, with cartilage and/or mucous glands in the airway wall, are histologically similar to bronchi. The term "medium airway" was used to emphasise the distal lung biopsy origin for airway tissue, but with a histological profile that was similar to proximal airways. Such classification of airways in TBBX was supported by distinct total inflammatory cell counts. Small airway cell counts were 3-12-fold higher than those recorded in the corresponding large or medium airways, where the cell counts were surprisingly similar to each other. When all lung regions were compared, the small airway had the highest inflammatory cell count. Interestingly, the ratio of individual inflammatory cell types was similar among all lung regions, except for the expected increased percentage of monocytes/ macrophages in alveolar tissue.

As a reference tissue for the density of inflammatory cells in small airways from TBBX a total of 12 small airways were evaluated from STS. Cross-sections of multiple well-defined small airways were available for analysis in each of three subjects with STS. Unfortunately, direct statistical comparison of small airways from TBBX with those in STS was not possible due to the inevitably small number of asthmatics with STS and the inherent heterogeneity of the disease. Nevertheless, the observation that the cell counts in the small airways from TBBX and STS fall within the same range, suggests that they represent a similar lung region.

In this study, it cannot be excluded that small airway cell counts were relatively higher because inhaled steroids (taken by 11 out of 14 subjects) decreased the large airway cell counts. However, when comparing large airway cell count data from normal subjects, mild, moderate and severe asthmatics (from 76 subjects in total, stored in a large database in the laboratory), severe asthmatics tended to have higher rather than lower large airway cell counts as compared to the other groups (data not shown). A formal TBBX study before and after inhaled steroids would be required to prove this concept.

It is important to expand studies of asthma to the distal lung. Although small, pathological studies of asthmatics suggest that inflammatory and/or structural changes in the small airways could influence asthma pathophysiology [2-9], studies to confirm or deny that possibility are nonexistent. An increasing proportion of the population suffers from this disorder and treatment remains inadequate in many cases. A better understanding of the nature and extent of their disease, as obtained from transbronchial biopsies, could eventually result in improvement in treatment. If disease is present and clinically important in the small airways and lung parenchyma, then improving delivery of medications to these regions of the lung, either through better aerosol or systemic delivery, could improve long-term outcomes.
Unfortunately, with new invasive types of research in a disease like asthma, the paradox that the invasiveness of the procedure limits the performance of the procedure must be dealt with, which is necessary before conclusions can be made on the usefulness of the procedure. In this study an average of 2.4 TBBXs per subject were obtained and $28 \%$ of these contained small airway tissue. Increased numbers of TBBXs per patient would likely result in a higher yield of small airway tissue. As suggested by Descombes et al. [11], five to six samples per subject would likely assure the presence of small airway tissue in at least one sample. However, as with the procedure itself, until the potential scientific usefulness of this procedure can be confirmed, the increased risk that accompanies the increased sampling is not likely to be accepted as a routine procedure in asthma studies.

Transbronchial biopsy is not, however, without risks. Investigators at the National Jewish Medical and Research Center have performed $>90$ transbronchial biopsies in subjects with mild-to-severe asthma. There has been one pneumothorax, which was treated conservatively. While the risk of this procedure is greater than that of endobronchial biopsies, severe asthma remains a disease with a high degree of morbidity (and even mortality). The present authors believe that the increased risk of transbronchial biopsy is justified, particularly when these studies are carried out at centres with a record of success in invasive studies and when the subjects are clearly informed of the risk of the intervention. Accordingly, transbronchial biopsy procedures should only be performed by experienced investigators, in a research setting until they are proven to be helpful in asthma studies.

In conclusion, the results of this study suggest that small airway tissue can be identified in transbronchial biopsy tissue samples and that it is histologically different from medium airway tissue also found in transbronchial biopsy tissue samples. Small airways from severe asthmatics have significantly higher numbers of inflammatory cells than medium airways or large airways. The higher numbers of inflammatory cells in small airways identified in transbronchial biopsy tissue samples are consistent with the cell numbers found in intact small airways identified in surgical tissue samples from this study and other studies [7, 19]. To assess the ultimate value of transbronchial biopsy for peripheral airway studies in asthma, more studies of transbronchial biopsy tissue samples in mild and moderate, as well as in severe asthmatics are necessary.

\section{References}

1. Laitinen LA, Laitinen A, Haahtela T. Airway mucosal inflammation even in patients with newly diagnosed asthma. Am Rev Respir Dis 1993; 147: 697-704.

2. Wenzel SE, Szefler SJ, Leung DY, Sloan SI, Rex MD, Martin RJ. Bronchoscopic evaluation of severe asthma. Persistent inflammation associated with high dose glucocorticoids. Am J Respir Crit Care Med 1997; 156: 737-743.

3. Saetta M, Di Stefano A, Rosina C, Thiene G, Fabbri 
LM. Quantitative structural analysis of peripheral airways and arteries in sudden fatal asthma. Am Rev Respir Dis 1991; 143: 138-143.

4. Synek M, Beasley R, Frew AJ, et al. Cellular infiltration of the airways in asthma of varying severity. $A m \mathrm{~J}$ Respir Crit Care Med 1996; 154: 224-230.

5. Kraft M, Djukanovic R, Wilson S, Holgate ST, Martin RJ. Alveolar tissue inflammation in asthma. Am J Respir Crit Care Med 1996; 154: 1505-1510.

6. Carroll N, Cooke C, James A. The distribution of eosinophils and lymphocytes in the large and small airways of asthmatics. Eur Respir J 1997; 10: 292-300.

7. Hamid Q, Song Y, Kotsimbos TC, et al. Respiratory pathophysiologic responses: inflammation of small airways in asthma. J Allergy Clin Immunol 1997; 100: 44-51.

8. Haley KJ, Sunday ME, Wiggs BR, et al. Inflammatory cell distribution within and along asthmatic airways. Am J Respir Crit Care Med 1998; 158: 565-572.

9. Kraft M, Martin RJ, Wilson S, Djukanovic R, Holgate ST. Lymphocyte and eosinophil influx into alveolar tissue in nocturnal asthma. Am J Respir Crit Care Med 1999; 159: 228-234.

10. Hernandez Borge J, Alfageme Michavila I, Munoz Mendez J, Villagomez Cerrato R, Campos Rodriguez F, Pena Grinan N. Factors related to diagnostic yield and complications of transbronchial biopsy. Arch Broncopneumol 1998; 34: 133-141.

11. Descombes E, Gardiol D, Leuenberger P. Transbronchial lung biopsy: an analysis of 530 cases with reference to the number of samples. Mon Arch Chest Dis 1997; 52: 324-329.
12. Rao VK, Ritter J, Kollef MH. Utility of transbronchial biopsy in patients with acute respiratory failure: a postmortem study. Chest 1998; 114: 549-555.

13. Papiris SA, Maniati M, Constantopoulos SH, Roussos C, Moutsopoulos HM, Skopouli FN. Lung involvement in primary Sjogren's syndrome is mainly related to the small airway disease. Ann Rheum Dis 1999; 58: 61-64.

14. Milne DS, Gascoigne AD, Coaker J, et al. Mononuclear phagocyte populations in the transplanted human lung. Transplantation 1998; 66: 671-673.

15. Nagata N, Takayama K, Nikaido Y, Yokosaki Y, Kido M. Comparison of alveolar septal inflammation to bronchoalveolar lavage in interstitial lung diseases. Respiration 1996; 63: 94-99.

16. Wenzel SE, Schwartz LB, Langmack EL, et al. Evidence that severe asthma can be divided pathologically into two inflammatory subtypes with distinct physiologic and clinical characteristics. Am J Respir Crit Care Med 1999; 160: 1001-1008.

17. Chu HW, Halliday JL, Martin RJ, Leung DY, Szefler SJ, Wenzel SE. Collagen deposition in large airways may not differentiate severe asthma from milder forms of the disease. Am J Respir Crit Care Med 1998; 158: 1936-1944.

18. Toothaker LE. Multiple comparisons for researchers. Newbury Park, NJ, Sage Publications, 1991; pp. 4142.

19. Shijubo N, Itoh Y, Yamaguchi T, et al. Clara cell protein-positive epithelial cells are reduced in small airways of asthmatics. Am J Respir Crit Care Med 1999; 160: 930-933. 